Article

\title{
On the Rehydration of Organic Layered Double Hydroxides to form Low-Ordered Carbon/LDH Nanocomposites
}

\author{
Eleonora Conterosito ${ }^{1}$, Luca Palin ${ }^{1,2}$, Diego Antonioli ${ }^{1}$, Maria Pia Riccardi ${ }^{3}$, Enrico Boccaleri ${ }^{1}$, \\ Maurizio Aceto ${ }^{1}$ (D), Marco Milanesio ${ }^{1, *}$ (I) and Valentina Gianotti $1, *$ (I) \\ 1 Dipartimento di Scienze e Innovazione Tecnologica, Università del Piemonte Orientale “A. \\ Avogadro" (Italy), Via Michel 11, I-15121 Alessandria, Italy; eleonora.conterosito@uniupo.it (E.C.); \\ luca.palin@uniupo.it (L.P.); diego.antonioli@uniupo.it (D.A.); enrico.boccaleri@uniupo.it (E.B.); \\ maurizio.aceto@uniupo.it (M.A.) \\ 2 Nova Res s.r.l., Via Dolores Bello 3, 28100 Novara, Italy \\ 3 Department of Earth and Environmental Sciences, University of Pavia, Via Ferrata 7, 27100 Pavia, Italy; \\ mariapia.riccardi@unipv.it \\ * Correspondence: marco.milanesio@uniupo.it (M.M.); valentina.gianotti@uniupo.it (V.G.); \\ Tel.: +39-0131-360226 (M.M.); +39-0131-360271 (V.G.)
}

Received: 22 July 2018; Accepted: 9 August 2018; Published: 14 August 2018

\begin{abstract}
Low-ordered carbon/layered double hydroxide (LDH) nanocomposites were prepared by rehydration of the oxides produced by calcination of an organic LDH. While the memory effect is a widely recognized effect on oxides produced by inorganic $\mathrm{LDH}$, it is unprecedented from the calcination/rehydration of organic ones. Different temperatures $\left(400,600\right.$, and $\left.1100{ }^{\circ} \mathrm{C}\right)$ were tested on the basis of thermogravimetric data. Water, instead of a carbonate solution, was used for the rehydration, with $\mathrm{CO}_{2}$ available from water itself and/or air to induce a slower process with an easier and better intercalation of the carbonaceous species. The samples were characterized by X-ray powder diffraction (XRPD), infrared in reflection mode (IR), and Raman spectroscopies and scanning electron microscopy (SEM). XRPD indicated the presence of carbonate LDH, and of residuals of unreacted oxides. IR confirmed that the prevailing anion is carbonate, coming from the water used for the rehydration and/or air. Raman data indicated the presence of low-ordered carbonaceous species moieties and SEM and XRPD the absence of separated bulky graphitic sheets, suggesting an intimate mixing of the low ordered carbonaceous phase with reconstructed LDH. Organic LDH gave better memory effect after calcination at $400{ }^{\circ} \mathrm{C}$. Conversely, the carbonaceous species are observed after rehydration of the sample calcined at $600{ }^{\circ} \mathrm{C}$ with a reduced memory effect, demonstrating the interference of the carbonaceous phase with $\mathrm{LDH}$ reconstruction and the bonding with LDH layers to form a low-ordered carbon/LDH nanocomposite.
\end{abstract}

Keywords: layered double hydroxides; graphene; graphite; low ordered carbon; mixed oxides; rehydration; memory effect; X-ray diffraction; Raman spectroscopy; scanning electron microscopy

\section{Introduction}

Layered double hydroxides (LDH) are materials that have attracted a lot of interest in the last few years [1-3] thanks to the possibility to act as a host for different inorganic or organic anions that can be intercalated between the mixed metals (e.g., $\mathrm{Zn} / \mathrm{Al}$ or $\mathrm{Mg} / \mathrm{Al}$ ) hydroxide layers to counterbalance the positive charge of the layer. The applications of layered double hydroxides span from catalysts [4-7] to host for drugs or cosmetics [8-13] to polymers additives to improve thermal stability and flame resistance [14-17] to adsorbents for decontamination [18-21]. Recently single-layer 
LDH nano-sheets applications in catalyzing oxygen evolution reactions and preparing light emitting devices, supercapacitors, and flame retardant nanocomposites were reviewed [22]. LDH colloids were also proposed as an alternative form for particular applications [23]. The preparation methods span from the traditional co-precipitation methods [24] to other more prone to scale up [3]. The exchange of the anion and the intercalation of organic anions can be achieved in different ways, which are covered extensively in the literature [3]. One of the methods used in particular for the intercalation of large organic anions is reconstruction upon hydration by exposure to air or to a solution containing the organic anion after the calcination of the inorganic LDH, exploiting the so-called "memory effect" [25] of LDH. The memory effect is an intriguing property of LDH and it consists of the ability of recovering the LDH structure after its collapse induced by thermal treatment [26-28]. The maximum temperature allowing the reconstruction of the structure depends on the LDH type, but is usually below $500{ }^{\circ} \mathrm{C}$ [29]. While there are many papers regarding the thermal characterization of the phase changes induced on inorganic LDH by thermal treatment [30-33] and their reconstruction [27-29,34], the number of studies on this aspect for hybrid LDH is rather limited [35-37]. The rehydration can be carried out by a carbonate solution [38] or deionized water with carbonate coming from $\mathrm{CO}_{2}$ in the water itself and/or air [39-41]. The rehydration is of course facilitated and is faster when carbonate is given in stoichiometric ratio or excess amounts in the solution used for the reconstruction.

In this work, we tried to investigate the lesser known part of the "memory effect", for example, the hydration of oxides obtained by calcination of organic LDH, with the goal of obtaining layered phases, mixed to low-ordered carbonaceous graphite-like species. Thermal treatments of both inorganic and organic LDH were carried out by a thermogravimetric analysis (TGA) experiment to have better control and to be able to track the weight loss. X-ray powder diffraction (XRPD) was used to assess the degree of reconstruction and infrared spectroscopy in reflectance mode (IR) to identify the anionic species. Finally, a Raman spectroscopy combined with the scanning electron microscopy study was performed on the carbon-containing LDH to confirm the presence of low-ordered carbonaceous graphite-like species, intimately mixed with LDH.

\section{Results}

\subsection{Sample Preparation and TGA and XRPD Analysis}

Two samples were chosen as staring materials for this study. The first sample is a hybrid $\mathrm{Zn} / \mathrm{Al}$ LDH intercalated with the NSAID drug Flurbiprofen (Flur) and obtained by liquid assisted grinding (LAG) method [11,42,43], and hereafter called LDH_Flur. The second sample is the $\mathrm{Zn} / \mathrm{Al}$ nitrate intercalated $\mathrm{LDH}$ used to prepare the hybrid sample, hereafter called $\mathrm{LDH} \_\mathrm{NO}_{3}$, as reference material with known "memory effect" properties. Figure 1 reports the XRPD patterns of the starting materials and of flurbiprofen alone (CCDC code FLUBIP) [44] as a reference, evidencing a high degree of crystallinity. In the LDH_Flur XRPD profile, a peak of residual $\mathrm{LDH}_{-} \mathrm{NO}_{3}$ (marked with a circle) and a peak due to a sodium nitrate residual (marked with an asterisk) is still visible because the XRPD pattern was collected before washing the sample. In the $\mathrm{LDH}_{-} \mathrm{NO}_{3}$ pattern, a broad and very weak peak not indexed by standard carbonate LDH structure can be seen at $2 \theta=7^{\circ}$, and one even weaker at $2 \theta=12.5^{\circ}$. Any attempts at assigning these two peaks with typical inorganic structures contaminating $\mathrm{LDH}$ or reactant/byproduct structure failed. As alternative way, attempts at fitting with one of the polytypes of $\mathrm{LDH}$ or with a supercell did not give convincing results; even if a supercell of standard hydrotalcite roughly fit the $2 \theta=7^{\circ}$ and $12.5^{\circ}$ peaks, a reliable assignation requires a far more detailed analysis beyond the scopes of the present paper. However, its elimination during the thermal treatment assures that this feature does not affect the rehydration process and, more importantly, these two peaks are not observed again after the LDH reconstruction. 


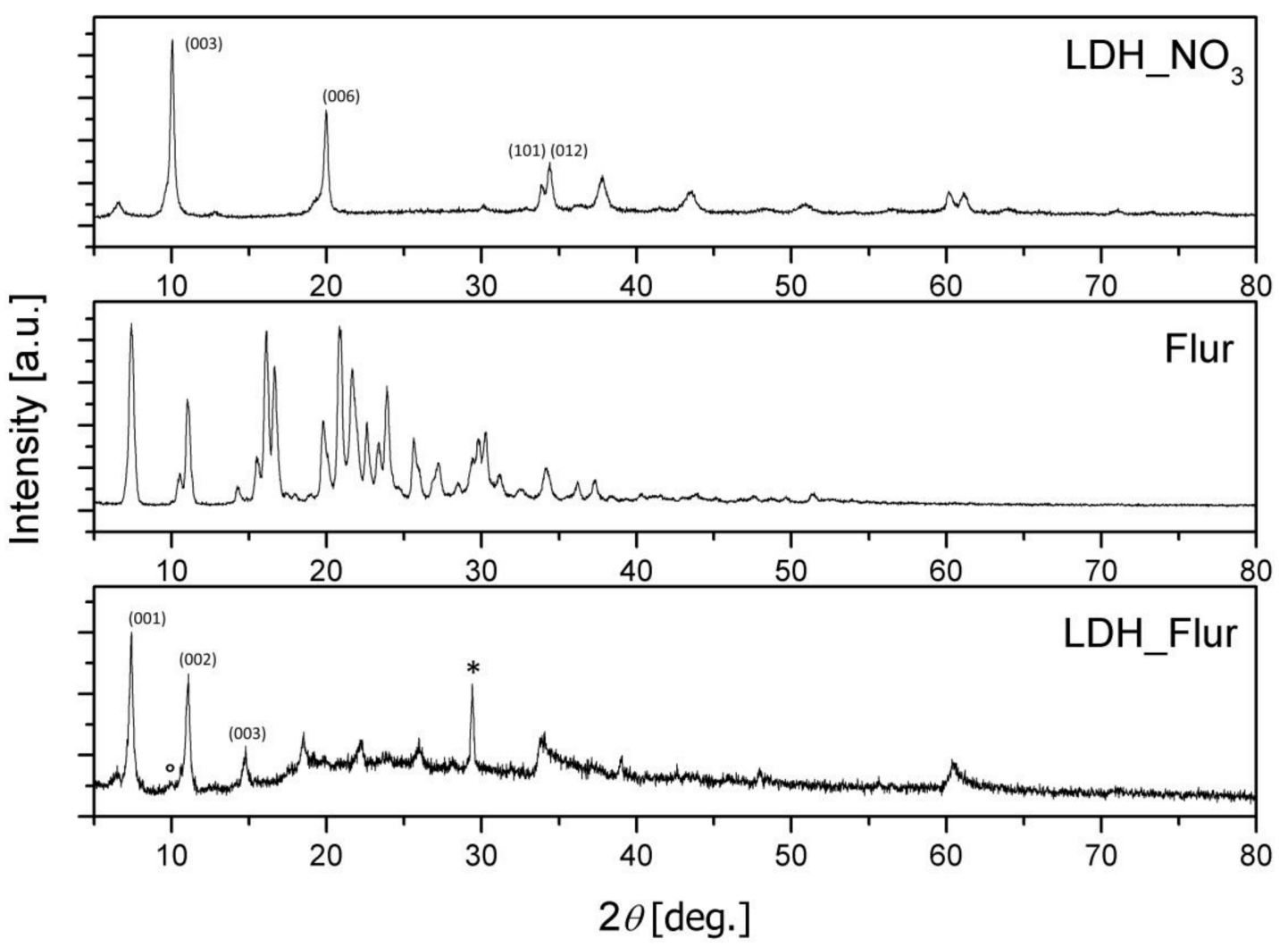

Figure 1. X-ray powder diffraction (XRPD) profiles of the starting samples. The indices are referred to as the layered double hydroxide (LDH) phases. In the LDH_Flurbiprofen (Flur) pattern, the circle marks the $\mathrm{LDH} \_\mathrm{NO}_{3}$ residue and the asterisk marks the $\mathrm{NaNO}_{3}$ residue.

A TGA analysis of the two starting samples was exploited to both identify the best temperature treatments and prepare the calcined samples. Preparing the samples used for the rehydration experiment by a TGA, equipped with an automatic sample holder, assured an identical treatment in the same inert atmosphere for every sample in Table 1, without any bias due to manual operator. It was also possible to reproduce the same heating conditions and atmosphere, with respect to a treatment in ovens or furnaces, whose temperature and atmosphere can not be controlled as finely as in the TGA furnace. Moreover, it was possible to follow the calcination and weight loss of the samples treated at the various temperatures (Figure 2). Three temperature were chosen; namely, (i) $400{ }^{\circ} \mathrm{C}$, when a first degradation occurred with a weight loss much smaller with respect to the organic content; (ii) $600{ }^{\circ} \mathrm{C}$, when the degradation of the organic is complete and only a residual carbonaceous phase should be present; and (iii) $1100^{\circ} \mathrm{C}$, when the degradation and elimination of organic moieties are complete.

The two samples used as starting materials and the three thermal treatments gave the six calcined samples, summarized in Table 1.

Table 1. Sample prepared by thermal treatment in the thermogravimetric analysis (TGA) furnace. LDH-layered double hydroxide; Flur-Flurbiprofen.

\begin{tabular}{|c|c|c|c|}
\hline \multirow{2}{*}{ Starting Sample } & \multicolumn{3}{|c|}{ Temp. Treatment $\left({ }^{\circ} \mathrm{C}\right)$} \\
\hline & 400 & 600 & 1100 \\
\hline $\mathrm{LDH} \_\mathrm{NO}_{3}$ & LDH_NO $3 @ 400$ & LDH_NO $3 @ 600$ & LDH_NO 301100 \\
\hline LDH_Flur & LDH_Flur@400 & LDH_Flur@600 & LDH_Flur@1100 \\
\hline
\end{tabular}




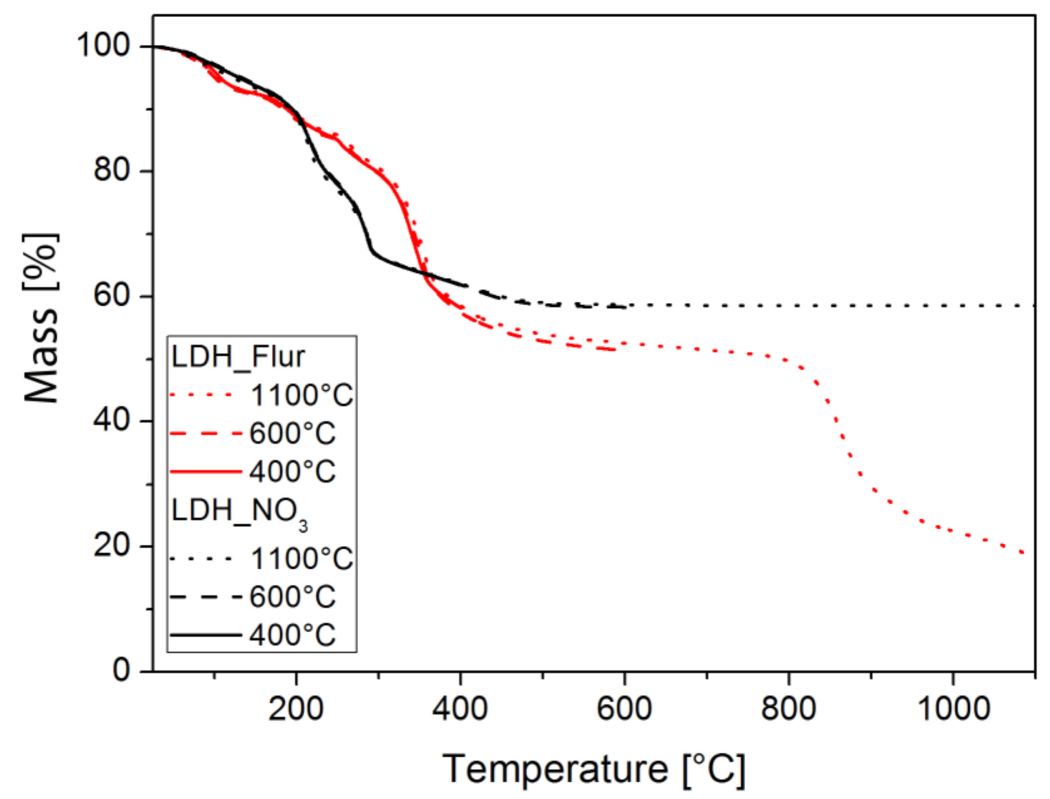

Figure 2. Thermogravimetric analysis (TGA) carried out to prepare the samples used for the characterization.

\subsection{Optimization of the Rehydration Protocol}

The rehydration was carried out on calcined samples using deionized water instead of a carbonate solution. In this case, carbonate is formed by adsorption of $\mathrm{CO}_{2}$ from the deionized water and/or the air and fixation. This choice, the alternative to using a carbonate solution, was done to induce a slower reconstruction process with an easier and better intercalation of the carbonaceous species within the layers. At first, XRPD was carried on the rehydrated samples to assess the degree of reconstruction, calculated by Rietveld refinement, using carbonate $\mathrm{LDH}$ and $\mathrm{ZnO}$ structures as detailed in the Supplementary Materials file. In this way, the various protocols used for the rehydration were tested and optimized. Two approaches were used, also to overcome the problem of the low wettability of the samples, being rather hydrophobic. The hydrophobicity is usually increased by the presence carbonaceous species, as already observed by Zhang et al. [45]. The combination of a slow rehydration mechanism with the hydrophobicity of the sample made the reaction rather slow and times up to 28 days were tested to obtain a good rehydration yield. In fact, typical times (hours) used for rehydration were not effective on carbonaceous-containing oxides and longer times were thus mandatory. A first method was a liquid assisted grinding (LAG)-like approach, similar to those used by some of us for anion exchange in hydrotalcites [42,43], consisting of mixing and gently grinding the sample and then adding a few drops of distilled water. In a series of preliminary measurements, the rehydration was repeated at intervals of hours and days, thus alternating hydration and ageing to develop and test the protocol. In a second approach, the hydration was carried out in a sealed vial to prevent evaporation. The evolution of the hydration protocol to arrive to the best one is described in the next subsections.

\subsubsection{Short-Time Hydration}

To assess the duration of the rehydration process, an in situ XRPD experiment was attempted by collecting one pattern, limited to the $9-14^{\circ} 2 \theta$ region, every one minute for one hour. These preliminary experiments demonstrated a very limited degree of reconstruction in the $\mathrm{LDH}_{-} \mathrm{NO}_{3} @ 600$ because the reconstruction is a slow process requiring days and not minutes or hours. In fact, when measuring in situ the rehydration of the $\mathrm{LDH}_{-} \mathrm{NO}_{3} @ 600$, a weak but still detectable peak (height of about 250 counts with oscillation of the background of around 120 counts) is observed (Figure 3). The same 
can be observed, even if with even weaker peaks in the other samples (Figure S2, see Supplementary Materials). This indicates that the rehydration starts just after wetting, but it is very slow and much longer times are needed, especially for organic-containing samples. Moreover, when measuring in situ in the open sample holder, evaporation is faster than rehydration, therefore, a way of minimizing evaporation was mandatory. To overcome the slowness of the reaction and the drying, in a second series of experiments, the samples were mixed and hydrated in a mortar, covered with parafilm, and then measured within 1 and $72 \mathrm{~h}$.

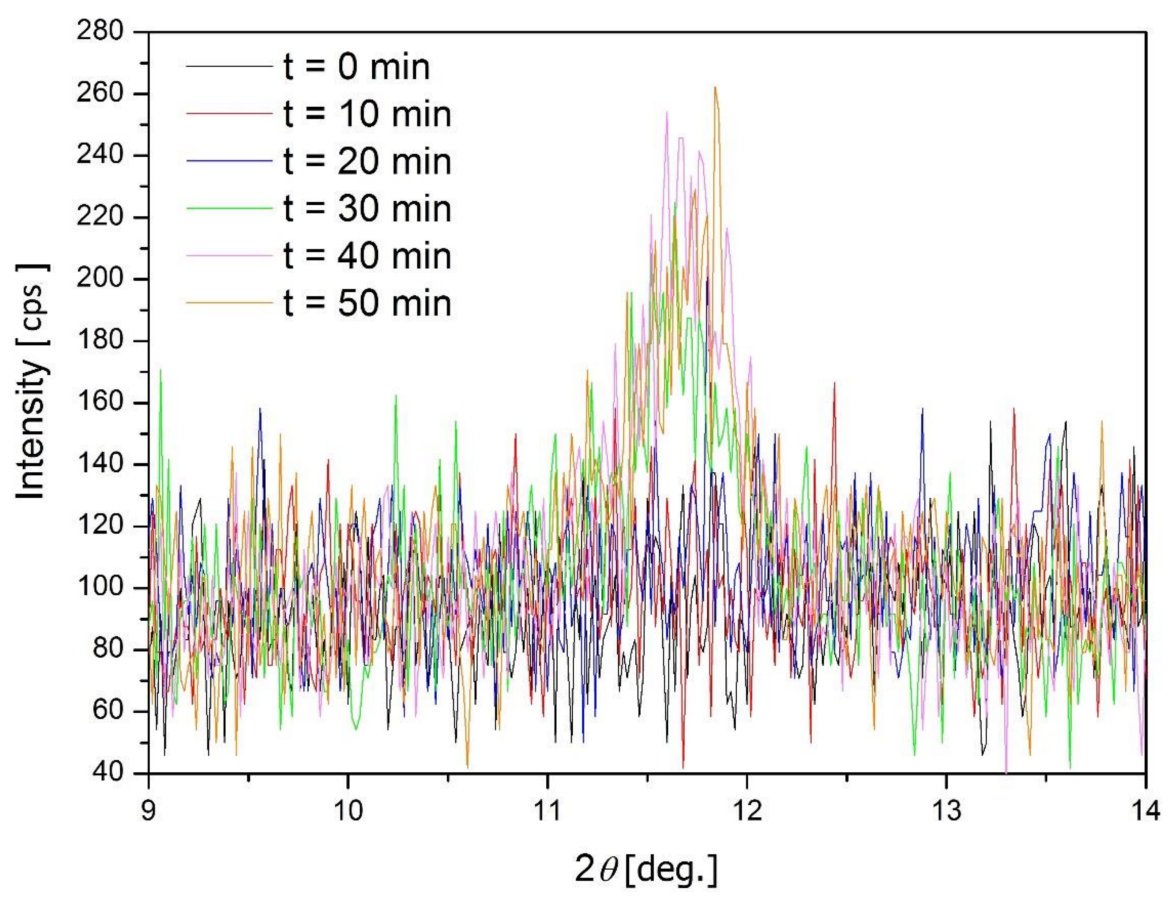

Figure 3. Selected patterns (one every $10 \mathrm{~min}$ ) from the in situ XRPD experiment on LDH_NO 30600 sample.

As a first indication, the reconstruction was unsuccessful, independent of the method, on the inorganic sample treated and $1100{ }^{\circ} \mathrm{C}$, and the residual mass after calcination at $1100{ }^{\circ} \mathrm{C}$ for the organic samples was a very small, difficult to manage amount. The organic samples at the highest temperatures were not characterized.

In the XRPD pattern of the $\mathrm{LDH}_{-} \mathrm{NO}_{3}$ sample calcined at $600{ }^{\circ} \mathrm{C}$ (Figure 4A), the broad band centered at about $23^{\circ}$ demonstrates the presence of an amorphous $\mathrm{Al}(\mathrm{OH})_{3}$ phase, while the sharp peaks correspond to crystalline $\mathrm{ZnO}$ (ICDD 75-0576).

It can be noticed that the basal 003 and 006, peaks of the LDH structure have appeared at $11.70^{\circ}$ and $23.48^{\circ}$ (Figure 4), respectively, but the peaks of $\mathrm{ZnO}$ are still present, indicating that the complete reconstruction or the LDH structure is not achieved after $24 \mathrm{~h}$ of rehydration (Figure $4 \mathrm{C}$ ). This is in agreement with Rocha et al. [29], stating that if the calcination temperature exceeds $550{ }^{\circ} \mathrm{C}$, more time is necessary to achieve full reconstruction. However, in this case, even after $72 \mathrm{~h}$, the reconstruction is not complete, as demonstrated by the presence of the mixed oxide peaks also in the XRPD pattern collected after $72 \mathrm{~h}$ (Figure 4D).

The in situ rehydration experiment of LDH_Flur@600 (Figure S2) was performed as described using the same protocol of the inorganic LDH. It is worth noting that in the XRPD pattern of LDH_Flur calcined at $600{ }^{\circ} \mathrm{C}$ (Figure $4 \mathrm{~A}$ ), a large diffraction bump between $12^{*}$ and $29^{\circ} 2 \theta$ is observed and attributed to amorphous $\mathrm{Al}(\mathrm{OH})_{3}$ and the $\mathrm{ZnO}$ peaks as in the $\mathrm{LDH} \mathrm{NO}_{3}$ sample, suggesting a rather different degree of order. After rehydration attempts, the appearance of the LDH peaks was not observed at any time, indicating that reconstruction did not occur in this case, as can be seen in 
Figure 5, where the XRPD patterns collected after $72 \mathrm{~h}$ are shown. This further confirms that oxides coming from calcination organic hydrotalcites are different, as already observed by XRPD, and more recalcitrant to rehydration. This is probably due to its increased hydrophobicity [45] with respect to fully inorganic ones, and a special and longer wetting protocol must be conceived.

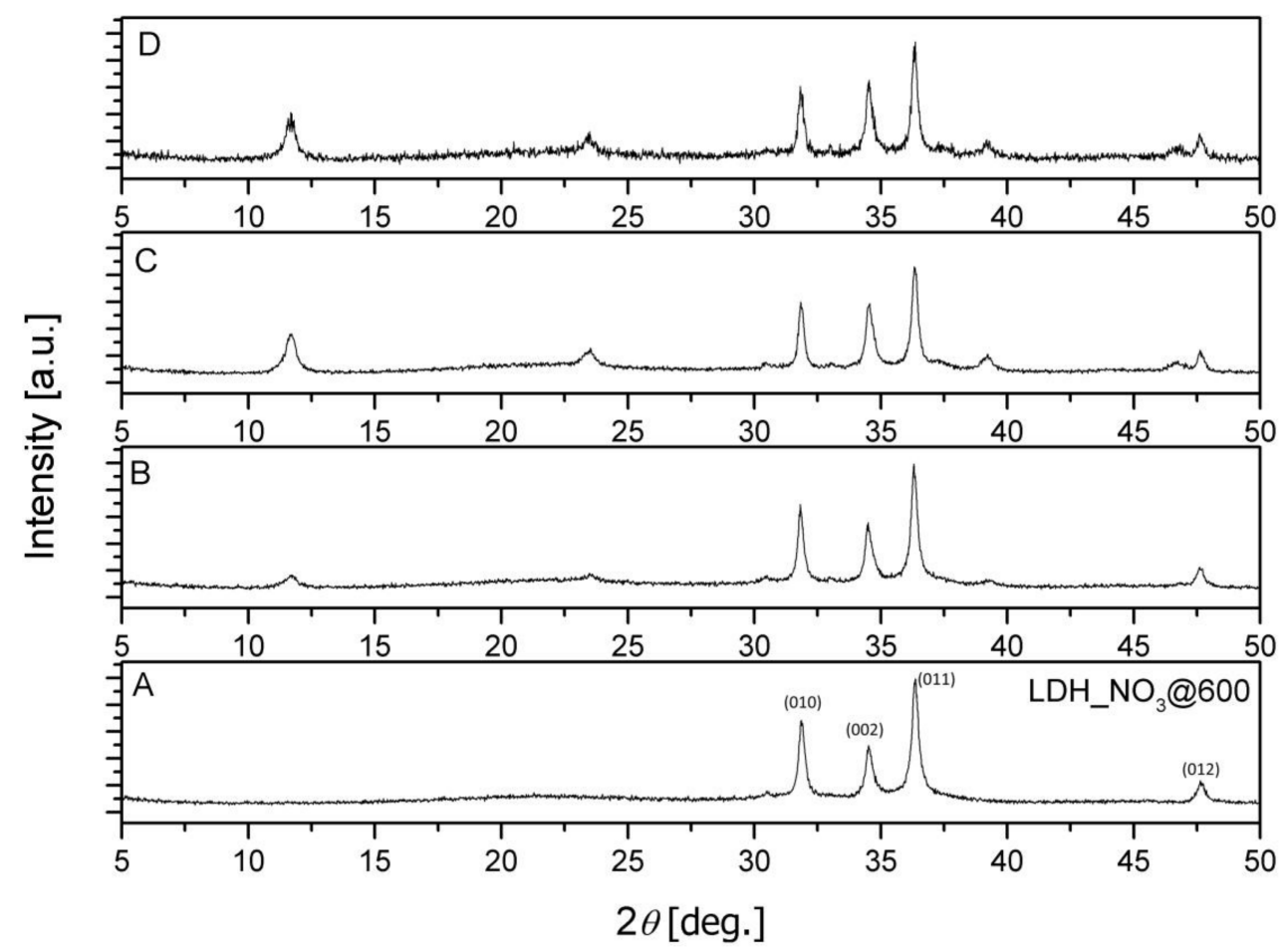

Figure 4. XRPD pattern of $\mathrm{LDH}_{-} \mathrm{NO}_{3}$ calcined at $600{ }^{\circ} \mathrm{C}(\mathrm{A})$; after $1 \mathrm{~h}$ rehydration $(\mathbf{B})$, after $24 \mathrm{~h}(\mathrm{C})$, and after $72 \mathrm{~h}(\mathbf{D})$. $\mathrm{ZnO}$ peaks are indexed in plot (A).

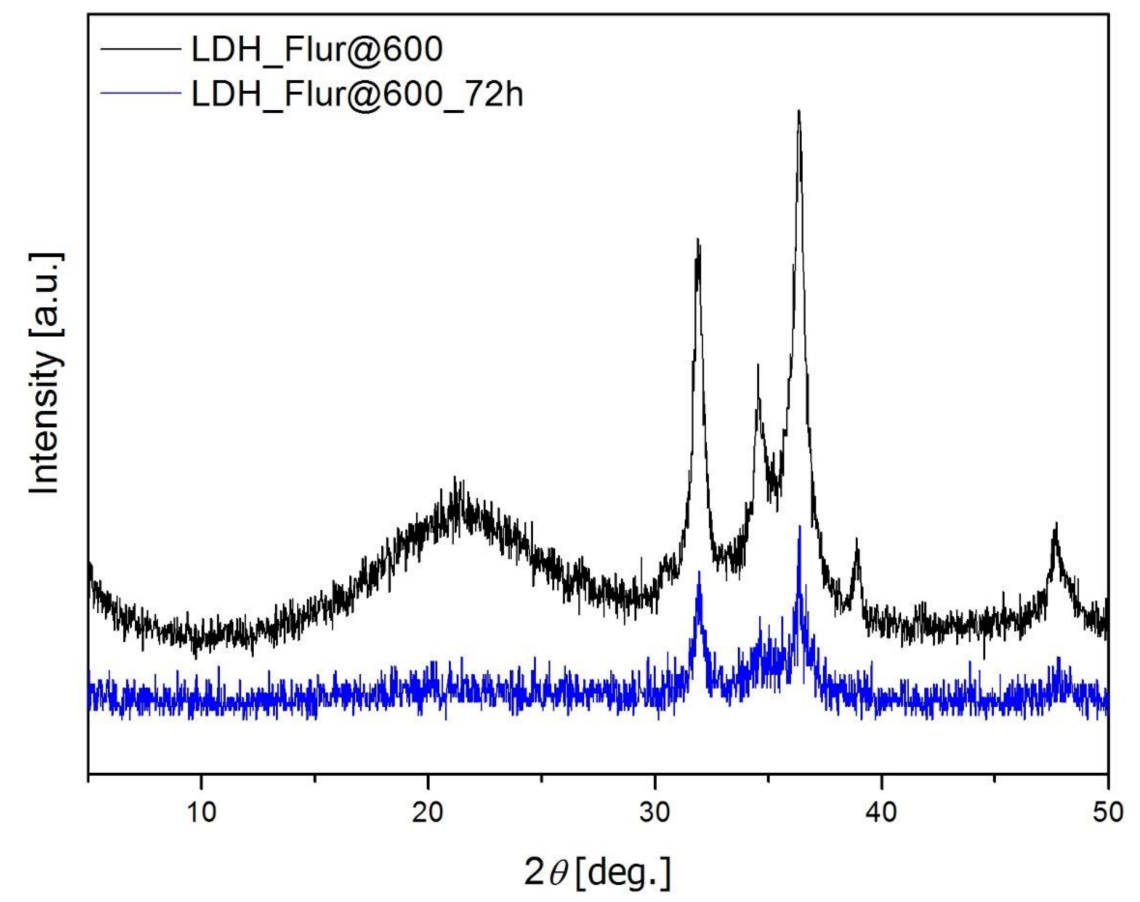

Figure 5. XRPD pattern of LDH_Flur calcined at $600{ }^{\circ} \mathrm{C}$ (blue) and after $72 \mathrm{~h}$ (black). 
Concerning the sample of LDH_Flur calcined at $1100{ }^{\circ} \mathrm{C}$, when analyzed by XRPD, it showed (Figure S3) the presence of the broad band of $\mathrm{Al}(\mathrm{OH})_{3}$ and the $\mathrm{ZnO}$ peaks. Moreover, a spinel $\mathrm{ZnAl}_{2} \mathrm{O}_{4}$ phase is present, matching the mineral Gahnite. The presence of this phase, typical of LDH calcined above $1000{ }^{\circ} \mathrm{C}$, is known to inhibit the reconstruction [29], and in fact, no change of the XRPD pattern occurs during the rehydration experiment. Therefore, the samples treated at $1100{ }^{\circ} \mathrm{C}$ were abandoned, concentrating the interest on $400{ }^{\circ} \mathrm{C}$ and $600{ }^{\circ} \mathrm{C}$ ones. Moreover, the hydration protocol was changed to allow a longer hydration, while preventing evaporation.

\subsubsection{Long-Time Hydration in a Vial}

A sealed vial was used to avoid drying and the time was increased to 14 days. Then, hydration was repeated for another 14 days for a total of 28 days, as detailed in the experimental section. The samples obtained by the longer rehydration in a sealed vial are listed in Table 2 . In this case, a good degree of reconstruction was obtained (Figure 6). Repeating the hydration helped obtaining a high reconstruction yield and increased crystallinity (see CS_L values obtained by Rietveld refinement of XRPD data in Table 3), especially in the case of the organic containing LDH.

The XRPD pattern obtained after rehydration of the inorganic samples (Figure 6 top) indicated that the reconstruction is already good after 14 days, and only a limited increase of the LDH peaks is observed after the second rehydration to arrive at 28 days of ageing. The $\mathrm{ZnO}$ oxide peaks are still present, evidencing a fraction recalcitrant to hydration also with this hydration protocol. In the organic LDH (Figure 6 bottom), the second rehydration gave an important increase of the LDH peaks, especially if the calcination was carried out at $600{ }^{\circ} \mathrm{C}$.
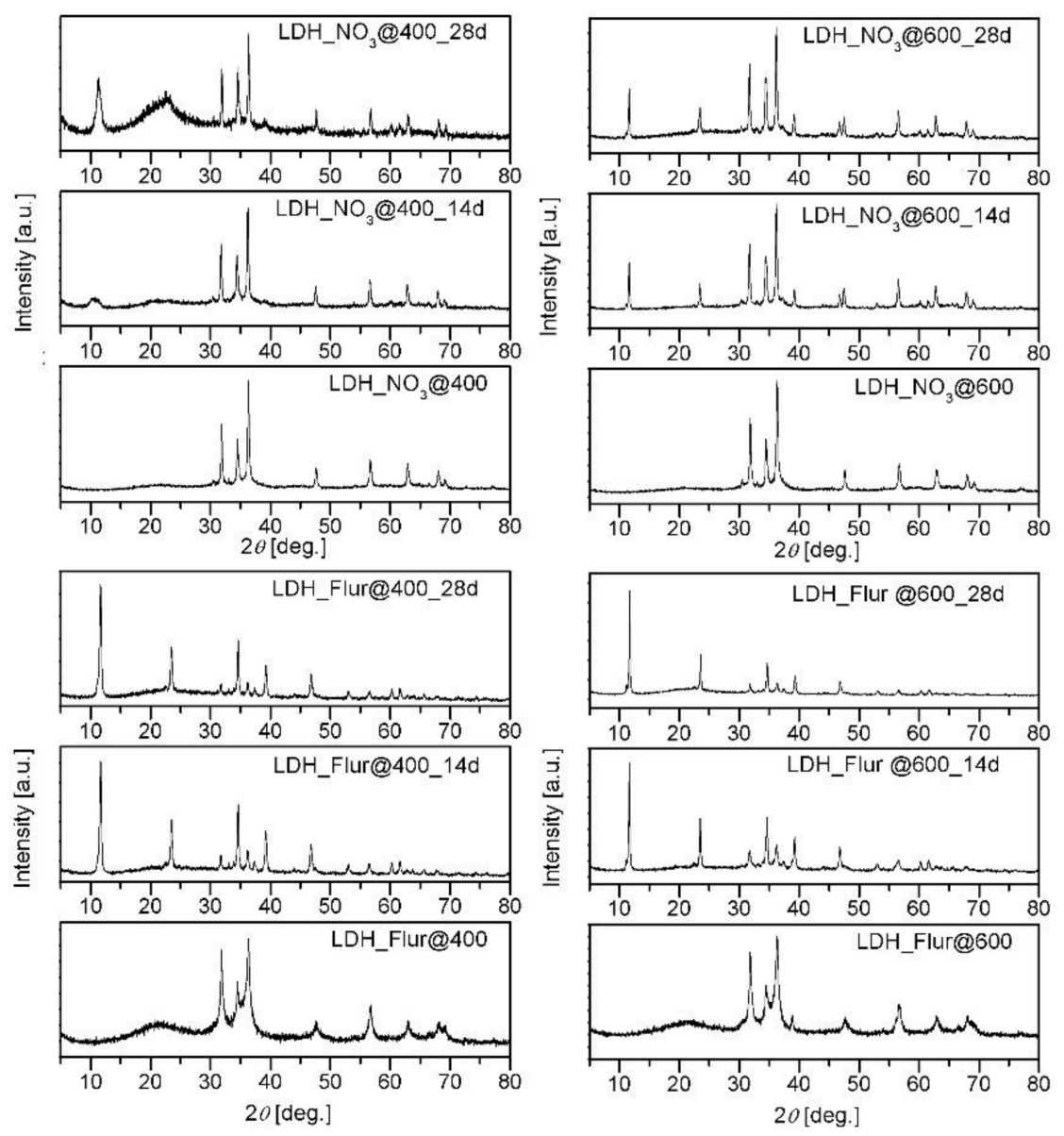

Figure 6. XRPD data before and after 14 and 28 days of ageing for the samples calcined at 400 and $600{ }^{\circ} \mathrm{C}$. 
Table 2. Summary of samples and treatments.

\begin{tabular}{|c|c|c|}
\hline \multirow{2}{*}{ Starting Sample } & \multicolumn{2}{|c|}{ Treatment Temperature $\left({ }^{\circ} \mathrm{C}\right)$} \\
\hline & 14 Days Hydration & 28 Days Hydration \\
\hline LDH_NO $3 @ 400$ & LDH_NO $3 @ 400 \_14 d$ & LDH_NO $3 @ 400 \_28 d$ \\
\hline LDH_Flur@400 & LDH_Flur@400_14d & LDH_Flur@400_28d \\
\hline LDH_NO3@600 & LDH_NO $3 @ 600 \_14 d$ & LDH_NO $3 @ 600 \_28 d$ \\
\hline LDH_Flur@600 & LDH_Flur@600_14d & LDH_Flur@600_28d \\
\hline
\end{tabular}

Table 3. Results of the Rietveld fit on the samples. The \% of the phases and c parameter of the LDH phases are reported. No error is given on phase weight \% because the e.s.d. (estimated standard deviation) calculated during Rietveld is much smaller than the realistic value. \% were therefore approximated to unit, the typical estimated precision of such measurements.

\begin{tabular}{ccccc}
\hline Samples & \% LDH_1 & \% ZnO & c LDH_1 (Å) & CS_L LDH_1 ${ }^{1}$ \\
\hline LDH_NO3@400_14d & 19 & 67 & $23.4(1)$ & 5 \\
LDH_NO3@400_28d & 60 & 40 & $23.6(7)$ & 8 \\
LDH_NO3@600_14d & 26 & 74 & $22.69(4)$ & 77 \\
LDH_NO3@600_28d & 31 & 69 & $22.69(4)$ & 78 \\
LDH_Flur@400_14d & 79 & 21 & $22.67(6)$ & 42 \\
LDH_Flur@400_28d & 85 & 15 & $22.67(6)$ & 42 \\
LDH_Flur@600_14d & 65 & 35 & $22.68(8)$ & 66 \\
LDH_Flur@600_28d & 73 & 27 & $22.67(6)$ & 80 \\
\hline
\end{tabular}

${ }^{1}$ Crystallite size parameter (CS_L) of the main LDH phase. Larger values correspond to higher crystallinity.

To quantify the degree of rehydration, a Rietveld refinement was carried out (see Figures S5-12 and Table 3), while to further investigate the nature of the samples, a spectroscopic and microscopic investigation is carried out.

The Rietveld analyses confirm that the hydration of inorganic $\mathrm{LDH}_{-} \mathrm{NO}_{3}$ samples gave more crystalline LDH if they were calcined at $600{ }^{\circ} \mathrm{C}$ with respect to $400{ }^{\circ} \mathrm{C}$, even if the amount is larger at the lower temperature. Conversely, the hydration of the calcined LDH_Flur samples in both cases gave crystalline $\mathrm{LDH}$ after rehydration, and a larger amount of layered phases in the sample prepared at $400{ }^{\circ} \mathrm{C}$ (79 vs. $\left.72 \%\right)$. As the samples after 28 days of reconstruction gave a higher degree of reconstruction, spectroscopic and morphological characterizations were carried out only on these samples. It is moreover noticeable by looking at the XRPD patterns of wet samples (Figure S13), how the formation of a larger amount of amorphous phase is correlated to a higher degree of reconstruction when the sample is dried.

\subsection{Spectroscopic Characterization}

A reflectance micro-IR measurement (Figure 7) was carried out to assess the nature of the sample and in particular of the anion after the reconstruction. The optical inspection indicated the presence of black aggregates, as a result of carbonaceous species, in the samples prepared from the organic LDH, while the samples prepared from the inorganic LDH are white. All samples showed homogeneous morphologies at this magnification level. In all samples, an evident band at $\sim 1350 \mathrm{~cm}^{-1}$ was observed, indicating the presence of carbonate in the reconstructed materials. Therefore, we can conclude that the rehydration occurred absorbing carbon dioxide from both water and air, and converting it into carbonate, finally intercalated into LDH as interlayer anion. This confirms the great $\mathrm{CO}_{2}$ absorption capability of the prepared oxides, as well as those obtained from calcination of organic LDH. 

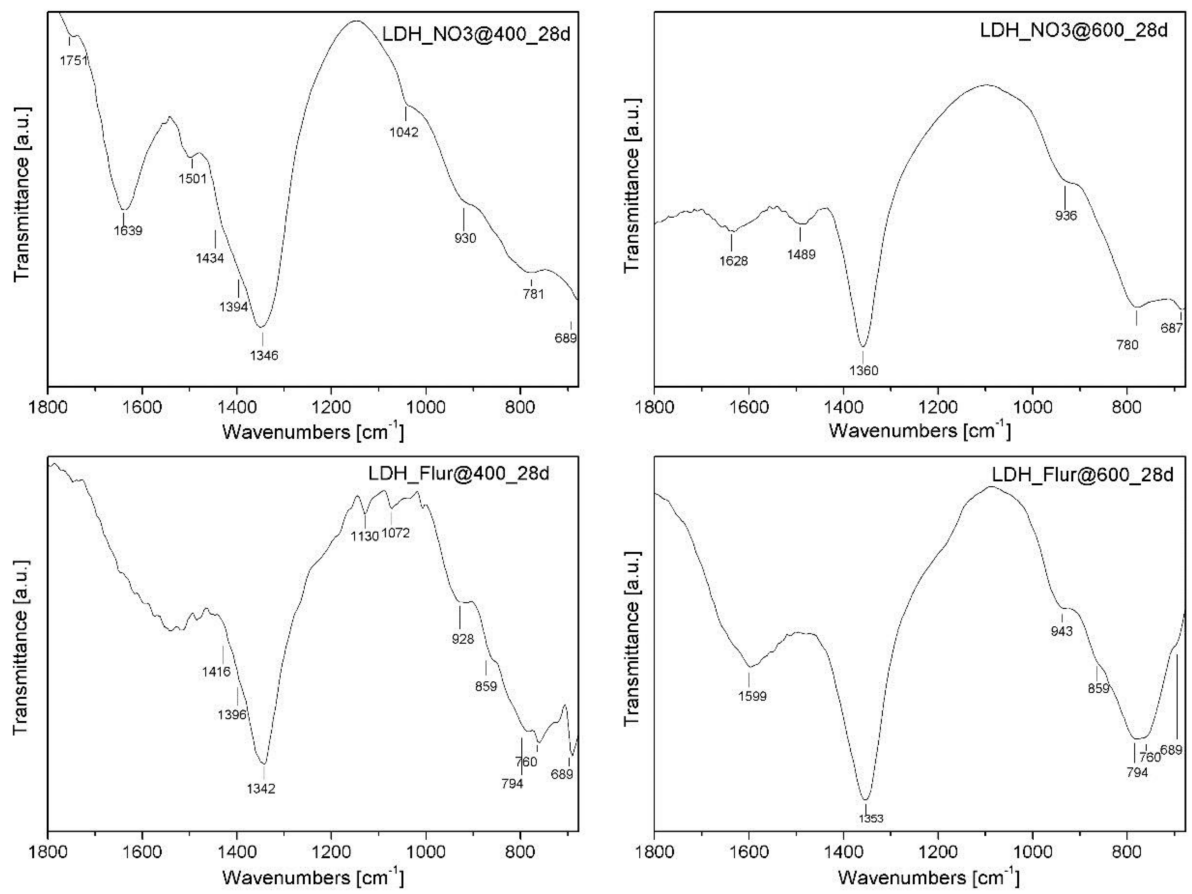

Figure 7. IR reflectance data on the samples after the samples after 28 days of reconstruction.

Raman spectroscopy (Figures 8 and 9) was employed to further investigate the conversion of species before and after the rehydration and the fate and conversion of the organic intercalated molecules into carbonaceous species. The laser of each experiment was chosen to minimize fluorescence and increase signal to noise ratio. In Figure 8, Raman spectra are collected with red laser $(633 \mathrm{~nm})$ for samples before hydration and green laser $(532 \mathrm{~nm})$ for hydrated samples.
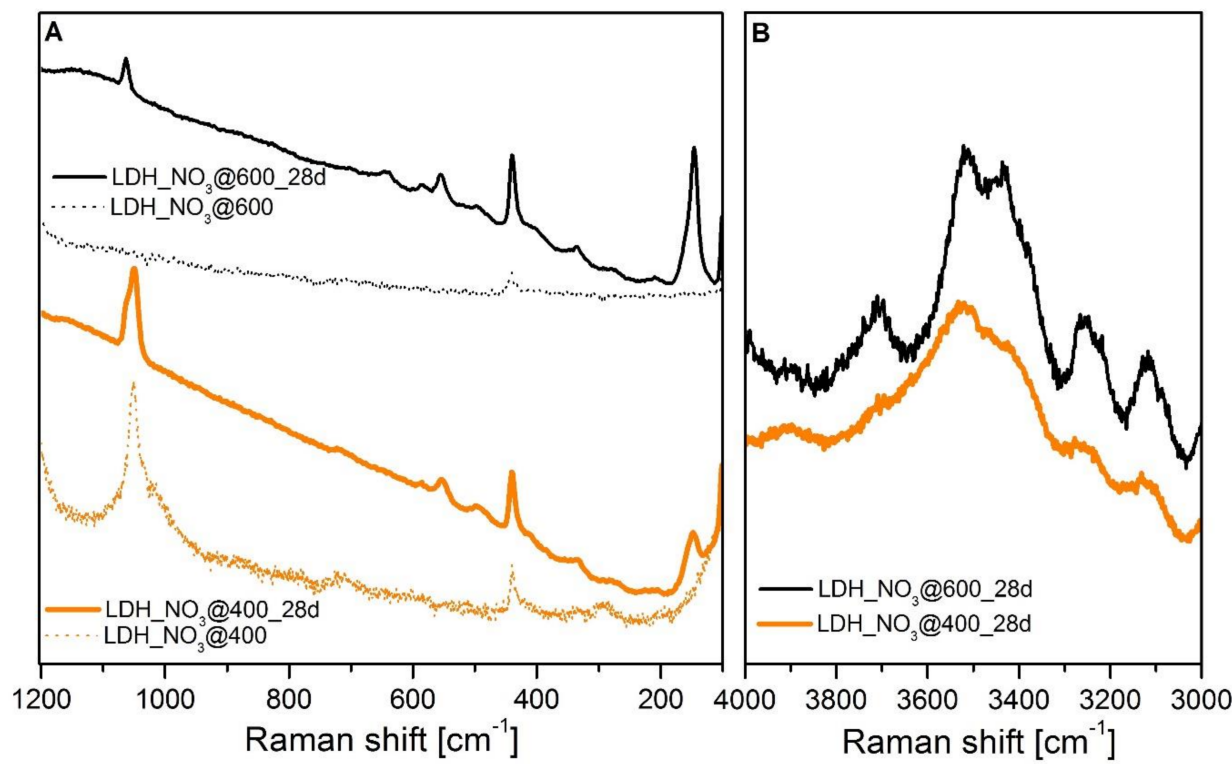

Figure 8. Raman data of $\mathrm{LDH} \_\mathrm{NO}_{3}$ after calcination at $400{ }^{\circ} \mathrm{C}$ (orange) and $600{ }^{\circ} \mathrm{C}$ (black) in the $1200-100 \mathrm{~cm}^{-1}$ range (A) and in the $4000-3000 \mathrm{~cm}^{-1}$ range (B) before rehydration (dotted lines) and after two hydrations and a total of 28 days of ageing (solid lines).

Considering the Raman spectra of thermally treated nitrate LDH (Figure 8A), before hydration, the sample treated at $400{ }^{\circ} \mathrm{C}$ highlights the presence of residual nitrate ions (featuring the band 
at $1050 \mathrm{~cm}^{-1}$ ) [31] and a signal around $440 \mathrm{~cm}^{-1}$ that, according to literature, can be related to $\mathrm{Zn}-\mathrm{O} / \mathrm{Zn}-\mathrm{OH}$ stretching modes [46]. Comparing the calcined samples at 400 and $600{ }^{\circ} \mathrm{C}$, the main evidence is the loss of the signal due to nitrate ions that appear to be completely removed after the treatment at higher temperatures. This is highlighted in the comparison of the spectra reported in Figure 8A.

Upon recovery of the structure due to hydration, two main contributions appear in the spectra of the samples treated at 400 and $600{ }^{\circ} \mathrm{C}$, respectively. The bands at low frequency (around 150, 440, 495, and 550 and minor signals between 250 and $350 \mathrm{~cm}^{-1}$ in Figure 8A) [47] of the sample treated at $400{ }^{\circ} \mathrm{C}$ are evidence of the recovery of hydroxylic moieties. The sample at $400{ }^{\circ} \mathrm{C}$ highlights the shouldering of the nitrate band by a component at higher frequency as a result of the formation of carbonate ions, with a typical Raman band at $1060 \mathrm{~cm}^{-1}$. The sample treated at $600{ }^{\circ} \mathrm{C}$ after recovery differs from the sample treated at $400{ }^{\circ} \mathrm{C}$ for the increase of the intensity of the layered structure vibrational features, but in particular for the signals related to the interlayer ions. In this case, the most evident aspect is the formation of $\mathrm{CO}_{3}{ }^{2-}$ anions as unique species, highlighted by the presence of the related band at $1060 \mathrm{~cm}^{-1}$. Comparing the spectra, Raman profiles confirm that the higher the temperature of treatment, the higher the extent of decomposition of nitrate ions and of the conversion of $\mathrm{LDH}$ to $\mathrm{ZnO} / \mathrm{Al}(\mathrm{OH})_{3}$ species, and the greater the effectiveness of the structural recovery. Further evidence between these two samples can be noted in the hydroxyl stretching region shown in Figure 8B, where samples treated at 400 and $600{ }^{\circ} \mathrm{C}$ reveal the gradual formation of the patterned profile reported in literature, consisting of three bands at around 3355-3360, 3440-3455, and 3535-3580 $\mathrm{cm}^{-1}$ [47].

Conversely, the samples originating from flurbiprofen-intercalated analogues (Figure 9) show only very weak signals coming from the inorganic fraction, close to the $300-500 \mathrm{~cm}^{-1}$ region (spectra of nitrate analogues are reported in Figure 8 for comparison). This weakness is due to the overwhelming absorption and fluorescence of the carbonaceous fraction with respect to the inorganic one. In this case, the presence of an organic molecule interleaving the hydroxide structure induces a fully different decomposition/reconstruction pathway. Upon thermal treatment, flurbiprofen is thermally decomposed to carbonaceous fractions (this occurs even at $400{ }^{\circ} \mathrm{C}$, where no signals of flurbiprofen features are found in the Raman and IR spectra). Weak broad signals around 1330 and $1590 \mathrm{~cm}^{-1}$ can be inferred, as witnesses of the beginning of formation of a low ordered carbonaceous species (Figure 9A,B, green curve) [46]. The extent and diffusion of this carbon-based moieties is highlighted by the $1300-1600 \mathrm{~cm}^{-1}$ region of the Raman spectrum of the sample treated at $600{ }^{\circ} \mathrm{C}$ (Figure 9A,B, blue curve). In this case, the spectral features in the interest regions reported above are far more evident and are consistent with low ordered carbonaceous structures, consistent with graphenic or multilayer carbonaceous sheets. The signals are observed clearly before (Figure 9A) and are still present, although weaker, after (Figure 9B) hydration. The presence of such carbonaceous phases is related to a relevantly different behavior of the inorganic $\mathrm{Zn} / \mathrm{Al}$ hydroxide layers. Raman spectroscopy confirms the presence of low ordered carbonaceous species, even if their location, within the layers of the LDH [48-50] or as separated entities, cannot be demonstrated by Raman spectroscopy only.

Scanning electron microscopy (SEM) was used to investigate the morphology of the samples and the location of carbonaceous species. At first, SEM micrograph confirms the larger crystallinity of LDH_NO3@600_28d sample (Figure 10B), where individual platelet-like crystallites are clearly observable, with respect to LDH_NO3@400_28d (Figure 10A), where aggregates of smaller crystallites are present, as already suggested by XRPD. The opposite is observed for the samples prepared starting from the organic sample (Figure 10 bottom). This indication confirms that the treatment at $400{ }^{\circ} \mathrm{C}$ (Figure 10C), with a limited production of carbonaceous species (as indicated by Raman spectroscopy), allows a better reconstruction, with features similar to those of inorganic samples treated at $600{ }^{\circ} \mathrm{C}$. In the sample treated at $600^{\circ}$ (Figure 10D), the presence of carbonaceous species induces a different reconstruction process, with much smaller crystallites. These indications, together with the absence of separated carbonaceous particles or graphitic species in the SEM analysis, strongly suggest that carbonaceous species are intimately mixed within the reconstructed LDH crystallites. 

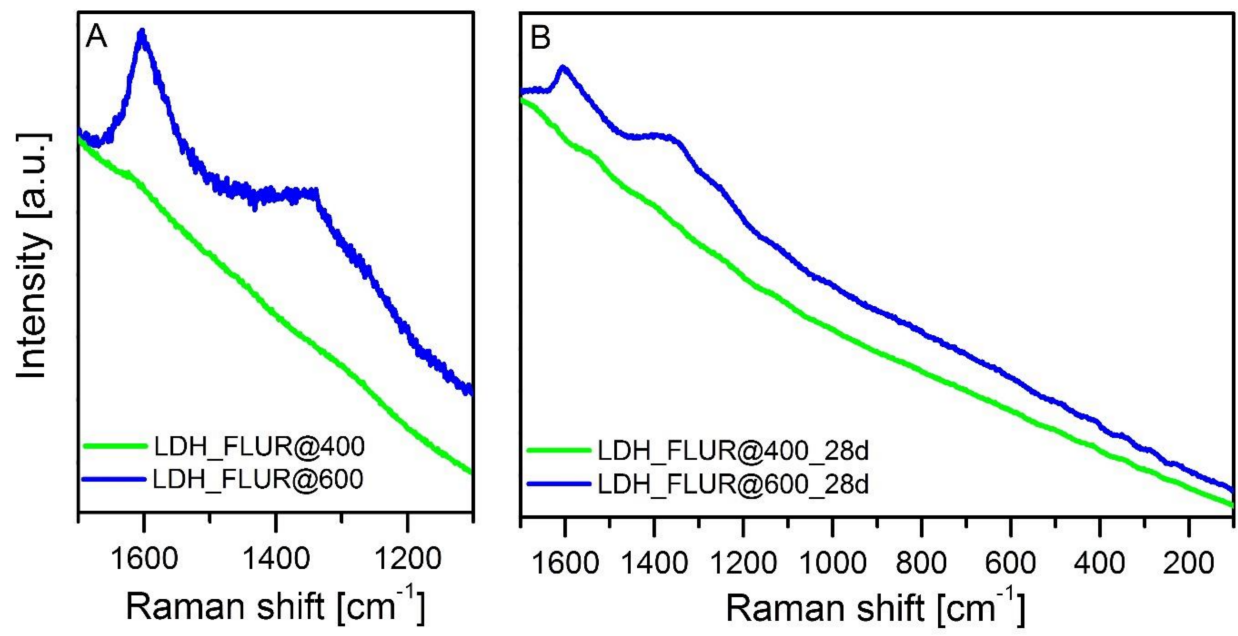

Figure 9. Raman data (green laser excitation) of LDH_FLUR after calcination at $400{ }^{\circ} \mathrm{C}$ (green) and $600{ }^{\circ} \mathrm{C}$ (blue) in the $1700-1100 \mathrm{~cm}^{-1}$ range before rehydration (A). Raman spectra in the range $1700-100 \mathrm{~cm}^{-1}$ of the FLUR samples after two hydrations and a total of 28 days of ageing (B).

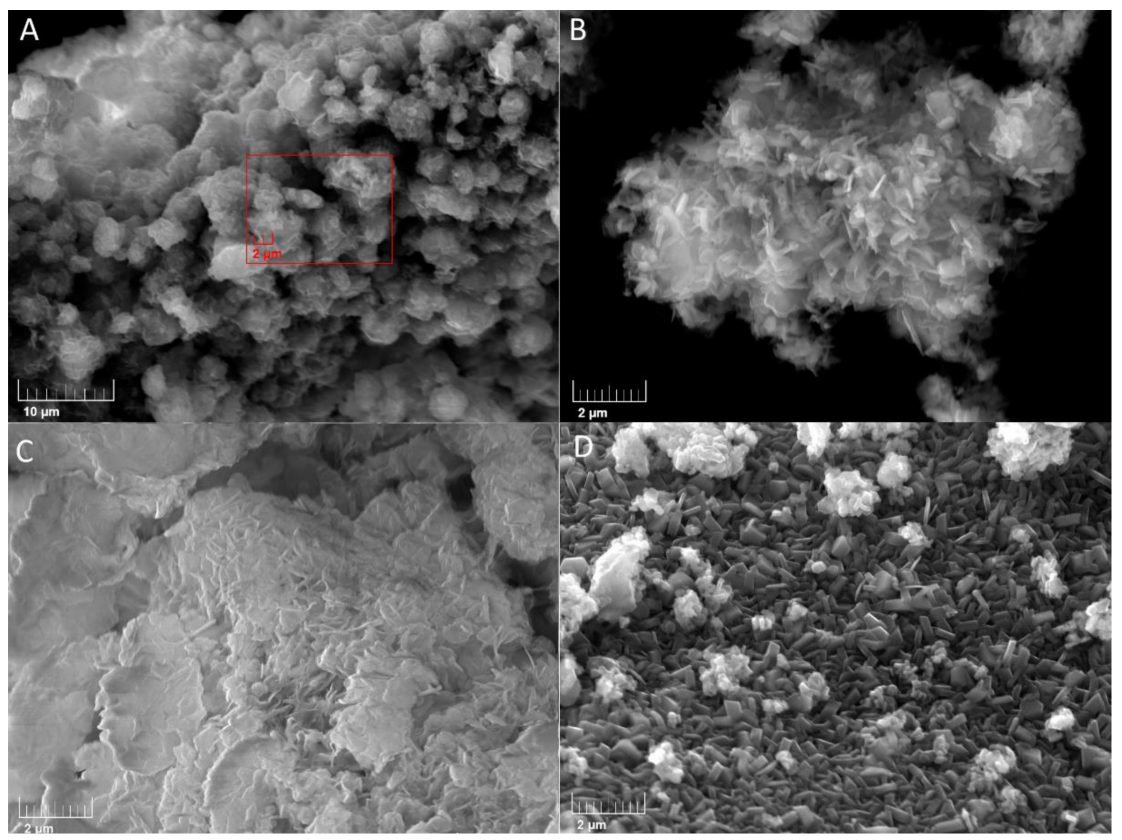

Figure 10. Scanning electron microscopy (SEM) images on the samples after 28 days of reconstruction. (A) LDH_NO 3 @400_28d (WD 9.30 mm, MAG 5.0k $\times$, view field (VF) $57.8 \mu \mathrm{m}$ ). The red box inserted to ease the comparison with the scale and view field of the other pictures. (B) LDH_NO3@600_28d (WD 9.34 mm, MAG 19.0k×, VF $15.2 \mu \mathrm{m}$ ); (C) LDH_Flur@400_28d (WD 9.32 mm, MAG 19.0k $\times$, VF $15.2 \mu \mathrm{m})$; (D) LDH_Flur@600_28d (WD 8.19 mm, MAG 19.0k×, VF $15.2 \mu \mathrm{m}$ ).

\section{Discussion}

The calcination and rehydration protocol of organic LDH was tested and optimized, while an inorganic LDH was used as reference. The process more efficient in giving reconstruction of carbon-containing LDH resulted rather slow, and the highest degree of reconstruction was obtained after a repeated hydration with deionized, but not de-carbonated water in a sealed vial, with a total of 28 days of ageing. Interestingly, the inorganic samples gave better reconstruction after calcination at $600{ }^{\circ} \mathrm{C}$, while the organic ones did so at $400{ }^{\circ} \mathrm{C}$. In the samples prepared starting from the flurbiprofen-containing samples, Raman data confirmed the presence of low ordered carbonaceous 
species, possibly including graphene, in the sample calcined at $600{ }^{\circ} \mathrm{C}$. The better reconstruction of the organic sample treated at $400{ }^{\circ} \mathrm{C}$ is ascribed to the limited production of carbonaceous species at $400{ }^{\circ} \mathrm{C}$, thus allowing a rehydration process similar to that observed for the inorganic samples. Conversely, the organic sample treated at $600{ }^{\circ} \mathrm{C}$, with a much larger amount of carbonaceous species, undergoes a different rehydration process, with smaller and more irregular observed crystallites. The absence of XRPD peaks due to graphitic and of graphenic phases in the SEM micrograph suggest that the low ordered carbonaceous species highlighted by Raman after the treatment at $600{ }^{\circ} \mathrm{C}$ are intimately mixed within LDH re-constructed species, to form a low-ordered carbon/LDH nanocomposite. The direct detection of single layer of graphene was indeed not obtained by SEM analysis, as the selected area electron diffraction investigations are necessary.

Concerning the mechanism of the reconstruction by the "memory effect" in the two cases (organic and inorganic LDH), the presence of carbonaceous species is able to interfere with the LDH reconstruction. A different decomposition mechanism can be inferred. In fact, in nitrate containing materials, the decomposition of nitrate promotes the presence of oxide ions and the reaction between atomic species on different layers, forming new crystalline phases. When the organic fraction is present, the process of charring increases the sample hydrophobicity and induce a lower wettability, thus preventing or reducing the formation of oxidic structures at low temperature and favoring amorphous hydroxides. This phenomenon prevents the interaction of the cationic species distributed on different lamellae of the LDH structure, inducing a markedly different morphology of the LDH reconstructed in presence of carbonaceous species. Finally, a nanocomposite, formed by carbonaceous species intimately mixed within the reconstructed LDH crystallites, is obtained.

\section{Materials and Methods}

\subsection{Sample Preparation}

$\mathrm{Zn} / \mathrm{Al} \mathrm{LDH} \_\mathrm{NO}_{3}$ prepared by urea method as described in the literature $[13,51]$ was used as starting material to prepare the LDH intercalated with Flurbiprofen (sample LDH_Flur), exploiting the liquid assisted grinding (LAG) method [42,43,52,53].

The calcination of the samples was obtained by thermal treatments imparted by TGA on LDH_Flur and on the LDH_NO ${ }_{3}$. The heating starts at $25^{\circ} \mathrm{C}$ and, by a heating rate of $10^{\circ} / \mathrm{min}$, reaches 400 or 600 or $1100{ }^{\circ} \mathrm{C}$ to obtain the samples LDH_Flur@400, LDH_Flur@600, LDH_Flur@1100 and LDH_NO 39400 , LDH_NO ${ }_{3} @ 600$, LDH_NO $3 @ 1100$, respectively.

The rehydration processes were carried out in two modes. The short-time hydration was performed mixing approximately $20 \mathrm{mg}$ of calcined sample for $3 \mathrm{~min}$ in a mortar with $60 \mu \mathrm{L}$ of deionized water, then the mortar was kept covered with parafilm for $72 \mathrm{~h}$. The XRPD characterizations were performed after $75 \mathrm{~min}$, and 24 and $72 \mathrm{~h}$. The long-time rehydration was performed by mixing $20 \mathrm{~g}$ of calcined sample for $3 \mathrm{~min}$ in a mortar with $60 \mu \mathrm{L}$ of deionized water, and then storing the sample in sealed vials to prevent evaporation. After 14 days, the samples were characterized by XRPD and then again put in contact with $60 \mu \mathrm{L}$ of deionized water and aged in a sealed vial for 14 days to reach a total of 28 days of ageing. These samples were characterized by XRPD, SEM, IR, and Raman spectroscopy.

\subsection{Instrumental Methods}

Thermogravimetric analysis (TGA) was performed using a thermo-balance TGA/SDTA LF1100 (Mettler-Toledo, Novate Milanese MI, Italy) at a scanning rate of $10^{\circ} \mathrm{C} / \mathrm{min}$ from room temperature to $1100{ }^{\circ} \mathrm{C}$ under $\mathrm{N}_{2}$.

Static X-ray measurements were performed on a ThermoARL powder diffractometer XTRA (ThermoFisher, Rodano, MI, Italy), equipped with a solid state Peltier cooled detector. All X-ray powder diffraction (XRPD) patterns were measured in continuous mode using the following conditions: $2 \theta$ angular range $5-80^{\circ}$, tube power $45 \mathrm{kV}$ and $40 \mathrm{~mA}$, step size $0.02^{\circ} 2 \theta$, integration time: $1.200 \mathrm{~s}$, 
scan rate: $1.000^{\circ} / \mathrm{min}$, in situ measures were performed in the range $9-14^{\circ} 2 \theta$ with a scan rate of $5.000^{\circ} / \mathrm{min}$ on the sample hydrated in the mortar as discussed in Section 4.1.

IR spectra were recorded on a Nicolet ${ }^{\mathrm{TM}} \mathrm{iN}^{\mathrm{TM}}$ iN10 infrared microscope (ThermoFisher, Rodano, MI, Italy) using the LN-cooled MCT detector to enhance sensitivity. Measurements were performed in reflection mode and then the plots were converted in \% transmittance to compare them with standard transmission IR spectra. Each spectrum is the average of 128 scans over an area of $150 \times 150 \mu \mathrm{m}$.

Raman spectra have been collected by different instrumentations equipped with different lasers and optics, to minimize the fluorescence problems on different samples and optimize signal detection for each sample.

The first was a FT-Raman RFS-100 instrument (Bruker, Milano, Italy) equipped with a $1.5 \mathrm{~W}$ $\mathrm{Nd}$ /YAG air-cooled laser delivering an excitation wavelength of $1064 \mathrm{~nm}$. The detector was a liquid nitrogen cooled germanium detector, allowing a spectral range 3500 to 50 (stokes) and -100 to $-2000 \mathrm{~cm}^{-1}$ (anti-stokes).

The second was a Renishaw inVia Reflex (Renishaw PLC, Gloucestershire, UK) micro-Raman spectrophotometer equipped with a cooled charge-coupled device camera at excitation wavelength of $514.5 \mathrm{~nm}$ with a laser power of $10 \mathrm{~mW}$ (spectral resolution and integration time of $3 \mathrm{~cm}^{-1}$ and $10 \mathrm{~s}$, respectively).

The third was a high-resolution dispersive Horiba Jobin Yvon (Villeneuve d'Ascq, France) Labram HRVIS model system, with an HR800 spectrometer, a confocal microscope, a $800 \mathrm{~mm}$ path monochromator, and a CCD cooled detector $\left(-70^{\circ} \mathrm{C}\right.$ ). $\mathrm{Nd}$ solid state green laser (wavelength $532 \mathrm{~nm}$, power $250 \mathrm{~mW}$ ) and 1800 lines $/ \mathrm{mm}$ grating were used, with a spectral resolution of $2 \mathrm{~cm}^{-1}$. Spectra have been taken placing the samples on the microscope stage and observing with long working distance $10 \times, 20 \times, 50 \times$, and $100 \times$ Olympus objectives. The sampled area was identified and focused using either a video camera or microscope binoculars. Spectra were obtained using laser at full power and checking visually with the camera view the absence of damages in the sampling area. Once optimized, spectra have been collected with variable exposure times (1 to $30 \mathrm{~s}$ ) or high number of acquisitions of short shots (10 acquisitions of $1 \mathrm{~s}$ then averaged), depending on the absolute counting given by the detector.

Raman spectroscopy and XRPD data were analyzed with OPUS [54] and TOPAS Academic v5.0 [55] software, respectively.

Textural and morphological observations (secondary electrons images-SE) were performed by means of a Tescan FE-SEM (Mira 3XMU-series), equipped with an EDAX energy-dispersive spectrometer. The operating conditions were as follows: $20 \mathrm{kV}$ accelerating voltage, around $13 \mathrm{~mA}$ beam current, different working distance and magnifications (reported in each photo). Samples for SEM observations were prepared either by C-coating from graphite evaporation.

Supplementary Materials: The following are available online at http:/ / www.mdpi.com/2304-6740/6/3/79/s1, Figure S1: Thermogravimetric analysis (TGA) plot (left) and first derivative (right) of the samples layered double hydroxide (LDH)_NO $\mathrm{NO}_{3}$ (black), Flurbiprofen (Flur) (red), LDH_Flur (green), and of the mechanical mixture of $\mathrm{LDH} \_\mathrm{NO}_{3}$ and Flurbiprofen (blue), Figure S2: X-ray powder diffraction (XRPD) patterns collected during the in situ rehydration experiments on LDH_FLUR@600 (A) and LDH_NO 3 @600 (B). Selection of one pattern every 10 min, Figure S3: Plot of the XRPD pattern of $\mathrm{LDH}_{-} \mathrm{NO}_{3}$ after calcination at $1100{ }^{\circ} \mathrm{C}$, Figure S4: Picture of the samples after calcination showing that the inorganic calcined LDHs remain white, while organic intercalated LDHs are black after the calcination, Figure S5: Plot of the Rietveld fit on the XRPD pattern of the LDH_FLUR@400_14d sample. Experimental patter in black, calculated curve in red. Peak tickmarks of the LDH phases in blue and black, zinc oxide in green, Figure S6: Plot of the Rietveld fit on the XRPD pattern of the LDH_FLUR@400_14d sample. Experimental patter in green, calculated curve in red. Peak tickmarks of the LDH phase in blue, zinc oxide in black, Figure S7: Plot of the Rietveld fit on the XRPD pattern of the LDH_FLUR@600_14d sample. Experimental patter in blue, calculated curve in red. Peak tickmarks of the LDH phase in blue, zinc oxide in black, Figure S8: Plot of the Rietveld fit on the XRPD pattern of the LDH_FLUR@600_28d sample. Experimental patter in green, calculated curve in red. Peak tickmarks of the LDH phase in blue, zinc oxide in black, Figure S9: Plot of the Rietveld fit on the XRPD pattern of LDH_NO $\mathrm{NO}_{3} @ 00 \_14 \mathrm{~d}$ sample. Experimental patter in brown, calculated curve in red. Peak tickmarks of the LDH phase in blue, zinc oxide in black, Figure S10: Plot of the Rietveld fit on the XRPD pattern of the LDH_Flur@400_28d sample. Experimental pattern in violet, calculated curve in red. Peak tickmarks of the LDH phase in blue, zinc oxide in black, Figure S11: Plot of the Rietveld 
fit on the XRPD pattern of LDH_Flur@600_14d sample. Experimental pattern in blue, calculated curve in red. Peak tickmarks of the LDH phase in blue, zinc oxide in black, Figure S12: Plot of the Rietveld fit on the XRPD pattern of LDH_Flur@600_28d sample. Experimental pattern in blue, calculated curve in red. Peak tickmarks of the LDH phase in blue, zinc oxide in black, Figure S13: Comparison of the XRPD patterns of the wet samples after 14 days of rehydration.

Author Contributions: Conceptualization, E.C. and M.M.; Data curation, L.P.; Formal analysis, E.C.; Investigation, E.C., L.P., D.A., M.P.R., E.B., M.A. and V.G.; Methodology, E.B., M.M. and V.G.; Supervision, M.M. and V.G.; Writing—original draft, E.C. and M.M.; Writing—review \& editing, V.G.

Acknowledgments: This research is original and had a financial support of the Università del Piemonte Orientale within the RICLOCK project.

Conflicts of Interest: The authors declare no conflict of interest.

\section{References}

1. Del Hoyo, C. Layered double hydroxides and human health: An overview. Appl. Clay Sci. 2007, 36, $103-121$. [CrossRef]

2. Duan, X.; Evans, D.G. Layered Double Hydroxides; Duan, X., Evans, D.G., Eds.; Structure and Bonding; Springer: Berlin/Heidelberg, Germany, 2006; Volume 119, ISBN 3-540-28279-3.

3. Conterosito, E.; Gianotti, V.; Palin, L.; Boccaleri, E.; Viterbo, D.; Milanesio, M. Facile preparation methods of hydrotalcite layered materials and their structural characterization by combined techniques. Inorg. Chim. Acta 2018, 470, 36-50. [CrossRef]

4. Cantrell, D.G.; Gillie, L.J.; Lee, A.F.; Wilson, K. Structure-reactivity correlations in MgAl hydrotalcite catalysts for biodiesel synthesis. Appl. Catal. A Gen. 2005, 287, 183-190. [CrossRef]

5. Sisani, M.; Costantino, U.; Vivani, R.; Nocchetti, M.; Costantino, F.; Montanari, F.; Cinotti, E. Hydrotalcite-Like Materials as Precursors of Catalysts for the Production of Hydrogen from Methanol. Available online: http://www.circc.uniba.it/conferenze/IV_PhD_ChemDay/Perugia_Sisani_Slides.pdf (accessed on 24 February 2007).

6. Othman, M.R.; Helwani, Z.; Fernando, W.J.N. Synthetic hydrotalcites from different routes and their application as catalysts and gas adsorbents: A review. Appl. Organomet. Chem. 2009, 23, 335-346. [CrossRef]

7. Ordóñez, S.; Díaz, E.; León, M.; Faba, L. Hydrotalcite-derived mixed oxides as catalysts for different C-C bond formation reactions from bioorganic materials. Catal. Today 2011, 167, 71-76. [CrossRef]

8. Perioli, L.; Ambrogi, V.; Rossi, C.; Latterini, L.; Nocchetti, M.; Costantino, U. Use of anionic clays for photoprotection and sunscreen photostability: Hydrotalcites and phenylbenzimidazole sulfonic acid. J. Phys. Chem. Solids 2006, 67, 1079-1083. [CrossRef]

9. Ambrogi, V.; Fardella, G.; Grandolini, G.; Perioli, L.; Tiralti, M.C. Intercalation compounds of hydrotalcite-like anionic clays with anti-inflammatory agents, II: Uptake of diclofenac for a controlled release formulation. AAPS PharmSciTech 2002, 3, 1-6. [CrossRef] [PubMed]

10. Ambrogi, V.; Fardella, G.; Grandolini, G.; Nocchetti, M.; Perioli, L. Effect of hydrotalcite-like compounds on the aqueous solubility of some poorly water-soluble drugs. J. Pharm. Sci. 2003, 92, 1407-1418. [CrossRef] [PubMed]

11. Perioli, L.; Ambrogi, V.; Nocchetti, M.; Rossi, C.; di Nauta, L. Effects of hydrotalcite-like nanostructured compounds on biopharmaceutical properties and release of BCS class II drugs: The case of flurbiprofen. Appl. Clay Sci. 2011, 51, 407-413. [CrossRef]

12. Perioli, L.; Ambrogi, V.; Bertini, B.; Ricci, M.; Nocchetti, M.; Latterini, L.; Rossi, C. Anionic clays for sunscreen agent safe use: Photoprotection, photostability and prevention of their skin penetration. Eur. J. Pharm. Biopharm. 2006, 62, 185-193. [CrossRef] [PubMed]

13. Costantino, U.; Ambrogi, V.; Nocchetti, M.; Perioli, L. Hydrotalcite-like compounds: Versatile layered hosts of molecular anions with biological activity. Microporous Mesoporous Mater. 2008, 107, 149-160. [CrossRef]

14. Yang, X.; Zhang, Q. Effect of hydrotalcite on the thermal stability, mechanical properties, rehology and fame retardance of poly(vinyl chloride). Polym. Int. 2004, 53, 698-707.

15. Liu, J.; Chen, G.; Yang, J. Preparation and characterization of poly(vinyl chloride)/layered double hydroxide nanocomposites with enhanced thermal stability. Polymer 2008, 49, 3923-3927. [CrossRef] 
16. Van der Ven, L.; van Gemert, M.L.; Batenburg, L.; Keern, J.; Gielgens, L.; Koster, T.P.; Fischer, H. On the action of hydrotalcite-like clay materials as stabilizers in polyvinylchloride. Appl. Clay Sci. 2000, 17, 25-34. [CrossRef]

17. Tong, M.; Chen, H.; Yang, Z.; Wen, R. The Effect of Zn-Al-Hydrotalcites Composited with Calcium Stearate and $\beta$-Diketone on the Thermal Stability of PVC. Int. J. Mol. Sci. 2011, 12, 1756-1766. [CrossRef] [PubMed]

18. Mohan, D.; Pittman, C.U. Arsenic removal from water/wastewater using adsorbents-A critical review. J. Hazard. Mater. 2007, 142, 1-53. [CrossRef] [PubMed]

19. Palmer, S.J.; Frost, R.L.; Nguyen, T. Hydrotalcites and their role in coordination of anions in Bayer liquors: Anion binding in layered double hydroxides. Coord. Chem. Rev. 2009, 253, 250-267. [CrossRef]

20. Kuzawa, K.; Jung, Y.J.; Kiso, Y.; Yamada, T.; Nagai, M.; Lee, T.G. Phosphate removal and recovery with a synthetic hydrotalcite as an adsorbent. Chemosphere 2006, 62, 45-52. [CrossRef] [PubMed]

21. Pavan, P.C.; Crepaldi, E.L.; Valim, J.B. Sorption of Anionic Surfactants on Layered Double Hydroxides. J. Colloid Interface Sci. 2000, 229, 346-352. [CrossRef] [PubMed]

22. Yu, J.; Wang, Q.; O'Hare, D.; Sun, L. Preparation of two dimensional layered double hydroxide nanosheets and their applications. Chem. Soc. Rev. 2017, 46, 5950-5974. [CrossRef] [PubMed]

23. Pavlovic, M.; Rouster, P.; Oncsik, T.; Szilagyi, I. Tuning Colloidal Stability of Layered Double Hydroxides: From Monovalent Ions to Polyelectrolytes. Chempluschem 2017, 82, 121-131. [CrossRef]

24. Theiss, F.L.; Ayoko, G.A.; Frost, R.L. Synthesis of layered double hydroxides containing $\mathrm{Mg}^{2+}, \mathrm{Zn}^{2+}, \mathrm{Ca}^{2+}$ and $\mathrm{Al}^{3+}$ layer cations by co-precipitation methods-A review. Appl. Surf. Sci. 2016, 383, 200-213. [CrossRef]

25. Cavani, F.; Trifirò, F.; Vaccari, A. Hydrotalcite-type anionic clays: Preparation, properties and applications. Catal. Today 1991, 11, 173-301. [CrossRef]

26. Sato, T.; Wakabayashi, T.; Shimada, M. Adsorption of various anions by magnesium aluminum oxide of $\left(\mathrm{Mg}_{0.7} \mathrm{Al}_{0.3} \mathrm{O}_{1.15}\right)$. Ind. Eng. Chem. Prod. Res. Dev. 1986, 25, 89-92. [CrossRef]

27. Roelofs, J.C.A.A.; van Bokhoven, J.A.; van Dillen, a.J.; Geus, J.W.; de Jong, K.P. The thermal decomposition of Mg-Al hydrotalcites: Effects of interlayer anions and characteristics of the final structure. Chem. Eur. J. 2002, 8, 5571-5579. [CrossRef]

28. Erickson, K.L.; Bostrom, T.E.; Frost, R.L. A study of structural memory effects in synthetic hydrotalcites using Environmental SEM. Mater. Lett. 2004, 59, 226-229. [CrossRef]

29. Rocha, J.; del Arco, M.; Rives, V.; Ulibarri, M.A. Reconstruction of layered double hydroxides from calcined precursors: A powder XRD and 27Al MAS NMR study. J. Mater. Chem. 1999, 3, 2499-2503. [CrossRef]

30. Kloprogge, J.T.; Frost, R.L. Infrared emission spectroscopic study of the thermal transformation of $\mathrm{Mg}^{-}, \mathrm{Ni}^{-}$ and Co-hydrotalcite catalysts. Appl. Catal. Gen. 1999, 184, 61-71. [CrossRef]

31. Kloprogge, J.T.; Wharton, D.; Hickey, L.; Frost, R.L. Infrared and Raman study of interlayer anions $\mathrm{CO}_{3}{ }^{2-}$, $\mathrm{NO}_{3}{ }^{-}, \mathrm{SO}_{4}{ }^{2-}$ and $\mathrm{ClO}_{4}{ }^{-}$in $\mathrm{Mg} / \mathrm{Al}-$ hydrotalcite. Am. Mineral. 2002, 87, 623-629. [CrossRef]

32. Kloprogge, J.T.; Kristof, J.; Frost, R.L. Thermogravimetric analysis-mass spectrometry (TGA-MS) of hydrotalcites containing $\mathrm{CO}_{3}{ }^{2-}, \mathrm{NO}_{3}{ }^{-}, \mathrm{Cl}^{-}, \mathrm{SO}_{4}{ }^{2-}$ or $\mathrm{ClO}_{4}{ }^{-}$. In Proceedings of the 12th International Clay Conference, A Clay Odyssey, Bahia-Blanca, Argentina, 22-28 July 2001; pp. 507-508.

33. Kloprogge, T.J.; Hickey, L.; Frost, R.L.; Kloprogge, J.T.; Hickey, L.; Frost, R.L. Heating stage Raman and infrared emission spectroscopic study of the dehydroxylation of synthetic Mg-hydrotalcite. Appl. Clay Sci. 2001, 18, 37-49. [CrossRef]

34. Wong, F.; Buchheit, R.G. Utilizing the structural memory effect of layered double hydroxides for sensing water uptake in organic coatings. Prog. Org. Coat. 2004, 51, 91-102. [CrossRef]

35. Conterosito, E.; Palin, L.; Antonioli, D.; Viterbo, D.; Mugnaioli, E.; Kolb, U.; Perioli, L.; Milanesio, M.; Gianotti, V. Structural Characterisation of Complex Layered Double Hydroxides and TGA-GC-MS Study on Thermal Response and Carbonate Contamination in Nitrate- and Organic-Exchanged Hydrotalcites. Chem. A Eur. J. 2015, 21, 14975-14986. [CrossRef] [PubMed]

36. Conterosito, E.; Croce, G.; Palin, L.; Pagano, C.; Perioli, L.; Viterbo, D.; Boccaleri, E.; Paul, G.; Milanesio, M. Structural characterization and thermal and chemical stability of bioactive molecule/hydrotalcite (LDH) nanocomposites. Phys. Chem. Chem. Phys. 2013, 15, 13418-13433. [CrossRef] [PubMed]

37. Sun, Y.; Pan, G.; Gu, Q.; Li, X.; Sun, G.; Ma, S.; Yang, X. Structural transformation and photoluminescence behavior during calcination of the layered europium-doped yttrium hydroxide intercalate with organic-sensitizer. Mater. Res. Bull. 2013, 48, 4460-4468. [CrossRef] 
38. Shiraga, M.; Kawabata, T.; Li, D.; Shishido, T.; Komaguchi, K.; Sano, T.; Takehira, K. Memory effect-enhanced catalytic ozonation of aqueous phenol and oxalic acid over supported $\mathrm{Cu}$ catalysts derived from hydrotalcite. Appl. Clay Sci. 2006, 33, 247-259. [CrossRef]

39. Miyata, S. Anion-exchange properties of hydrotalcite-like compounds. Clays Clay Miner. 1983, 31, $305-311$. [CrossRef]

40. Valente, J.S.; Figueras, F.; Gravelle, M.; Kumbhar, P.; Lopez, J.; Besse, J.-P. Basic Properties of the Mixed Oxides Obtained by Thermal Decomposition of Hydrotalcites Containing Different Metallic Compositions. J. Catal. 2000, 189, 370-381. [CrossRef]

41. Zhu, H.; Tang, P.; Feng, Y.; Wang, L.; Li, D. Intercalation of IR absorber into layered double hydroxides: Preparation, thermal stability and selective IR absorption. Mater. Res. Bull. 2012, 47, 532-536. [CrossRef]

42. Milanesio, M.; Conterosito, E.; Viterbo, D.; Perioli, L.; Croce, G. New Efficient Intercalation of Bioactive Molecules into Layered Double Hydroxide Materials by Solid-State Exchange: An in Situ XRPD Study. Cryst. Growth Des. 2010, 10, 4710-4712. [CrossRef]

43. Conterosito, E.; Van Beek, W.; Palin, L.; Croce, G.; Perioli, L.; Viterbo, D.; Gatti, G.; Milanesio, M. Development of a Fast and Clean Intercalation Method for Organic Molecules into Layered Double Hydroxides. Cryst. Growth Des. 2013, 13, 1162-1169. [CrossRef]

44. Flippen, J.L.; Gilardi, R.D. (+)-2-(2-Fluoro-4-biphenyl)propionic acid (flurbiprofen). Acta Crystallogr. Sect. B Struct. Crystallogr. Cryst. Chem. 1975, 31, 926-928. [CrossRef]

45. Zhang, Y.; Yu, P.; Qi, Y.; Chen, F.; Li, Y.; Zhang, Y. Oleylamine/graphene-modified hydrotalcite-based film on titanium alloys and its lubricating Properties Oleylamine/graphene-modified hydrotalcite-based film on titanium alloys and its lubricating properties. Mater. Lett. 2017, 193, 93-96. [CrossRef]

46. Boccaleri, E.; Arrais, A.; Frache, A.; Gianelli, W.; Fino, P.; Camino, G. Comprehensive spectral and instrumental approaches for the easy monitoring of features and purity of different carbon nanostructures for nanocomposite applications. Mater. Sci. Eng. B 2006, 131, 72-82. [CrossRef]

47. Kloprogge, J.T.; Hickey, L.; Frost, R.L. FT-Raman and FT-IR spectroscopic study of synthetic Mg/Zn/Al-hydrotalcites. J. Raman Spectrosc. 2004, 35, 967-974. [CrossRef]

48. Plank, J.; Zou, N.; Zhao, Z.; Dekany, I. Preparation and Properties of a Graphene Oxide Intercalation Compound Utilizing Hydrocalumite Layered Double Hydroxide as Host Structure. Z. Anorg. Allg. Chem. 2014, 640, 1413-1419. [CrossRef]

49. Lonkar, S.P.; Raquez, J.M.; Dubois, P. One-Pot Microwave-Assisted Synthesis of Graphene/Layered Double Hydroxide (LDH) Nanohybrids. Nano-Micro Lett. 2015, 7, 332-340. [CrossRef]

50. Li, M.; Zhu, J.E.; Zhang, L.; Chen, X.; Zhang, H.; Zhang, F.; Xu, S.; Evans, D.G. Facile synthesis of NiAl-layered double hydroxide/graphene hybrid with enhanced electrochemical properties for detection of dopamine. Nanoscale 2011, 3, 4240-4246. [CrossRef] [PubMed]

51. Costantino, U.; Nocchetti, M.; Vivani, R.; Marmottini, F. New synthetic routes to hydrotalcite-like compounds-Characterisation and properties of the obtained materials. Eur. J. Inorg. Chem. 1998, 1439-1446. [CrossRef]

52. Toson, V.; Conterosito, E.; Palin, L.; Boccaleri, E.; Milanesio, M.; Gianotti, V. Facile Intercalation of Organic Molecules into Hydrotalcites by Liquid-Assisted Grinding: Yield Optimization by a Chemometric Approach. Cryst. Growth Des. 2015, 15, 5368-5374. [CrossRef]

53. Conterosito, E.; Milanesio, M.; Palin, L.; Gianotti, V. Rationalization of liquid assisted grinding intercalation yields of organic molecules into layered double hydroxides by multivariate analysis. RSC Adv. 2016, 6, 108431-108439. [CrossRef]

54. Bruker Optik GmbH. OPUS, Version 5.5; Software Reference Manual; Bruker Optik GmbH: Ettligen, Germany, 2003.

55. Coelho Software. TOPAS-Academic, Version 5; General Profile and Structure Analysis Software for Powder Diffraction Data; Coelho Software: Brisbane, Australia, 2012.

(C) 2018 by the authors. Licensee MDPI, Basel, Switzerland. This article is an open access article distributed under the terms and conditions of the Creative Commons Attribution (CC BY) license (http:/ / creativecommons.org/licenses/by/4.0/). 